\title{
CARACTERIZAÇÃO DE MIOPATIAS MITOCONDRIAIS ATRAVÉS DA AVALIAÇÃO DAS ATIVIDADES ENZIMÁTICAS ENVOLVIDAS NO METABOLISMO ENERGÉTICO
}

\author{
FÁBIO CESAR PEDROSO*, ANNIBAL P. CAMPELLO*, \\ LINEU CESAR WERNECK **, MARIA LUUCIA W. KLÜPPEL*.
}

\begin{abstract}
RESUMO - Foi determinada a atividade das enzimas NADH desidrogenase, NADH citocromo c redutase, succinato desidrogenase, succinato citocromo $\mathrm{c}$ redutase, citocromo $\mathrm{c}$ oxidase e citrato sintase $\mathrm{em}$ mitocôndrias de músculo esquelético humano normal e doente (suspeito de miopatia mitocondrial). O grupo controle foi constituído de 13 indivíduos normais e que não faziam uso contínuo de fámacos. $\mathrm{O}$ grupo doente era constituído de 10 pacientes cujo diagnóstico anatomopatológico indicava suspeita de miopatia mitocondrial. Obscivout-se redução na atividade das enzimas em todos os pacientes: 7 com anormalidades em todas as enzimas ensaiadas: 2 com deficiências em todas as enzimas exceto na citocromo c oxidase; e 1 paciente com disfunçāo apenas na atividade da succinato desidrogenase e succinato citocromo c redutase. Este perfil possibilitou caracterizar múltiplas deficiências ou deficiência combinada da cadeia respiratória, além da disfunção na citrato sintasce em 9 pacientes. Um dos casos constituiu exceção, sendo a deficiência enzimática restrita ao complexo II. Foi possivel concluir que a metodologia usada é adequada c facilmente aplicável nos objetivos clínicos. Os resultados obtidos possibilitam a caracterização dos complexos enzimáticos mitocondriais deficientes, mostrando que tais enfermidades sño originadas de disfunção no metabolismo energético.
\end{abstract}

PALAVRAS-CHAVE: mitocôndria, músculo esquelético humano, miopatia mitocondrial, NADH desidrogenase, NADH citocrono $\mathrm{c}$ redutase, succinato desidrogenase, succinato citocromo $\mathrm{c}$ redutase, citocromo c oxidasc, citrato sintase.

Characterization of mitochondrial myopathies through the evaluation of the enzymatic activities involved in the energetic metabolism

ABSTRACT- The activities of the enzymes NADH dehydrogenase, NADH cytochrome c reductase, succinate dehydrogcnase, succinate cytochrome $c$ reductase, cytochrome $c$ oxidase and citrate synthase in normal and sick human skeletal muscle mitochondria were determined. A control group was formed by 13 normal people and without using continuous medication. The patient group was formed by 10 people whose pathological diagnosis indicated suspicion of milochondrial myopathy. A decrease in the activity of the enzymes in all patient was observed: 7 with abnormality in all the tested enzymes; 2 with deficiencies in all the elazymes except cytochrome $\mathrm{c}$ oxidase; and I with dysfunction only in the activities of succinite dehydrogenase and succinate cytochrome $\mathrm{c}$ reductase. The results indicate multiple or combined deficiencics in the respiratory chain, besides dysfunction of citrate synthase in 9 patients. In one exceptional case, the enzymatic deficiency was rustricted to complex II. It is possible to conclude that the methodology used herein is adequate and easily applicable to clinical objectives, and that the results obtained allow characterization of the deficient mitochondrial enzymatic complexes, thus showing that the origin of the diseases is an energetic metabolic dysfunction.

KEY WORDS: mitochondria human skeletal muscle, mitochondrial myopathy. NADH cytochrone c reductase, NADH dehydrogenase, succinate dehydrogenase, succinate cytochrome c reductasc, cytochromc $c$ oxidase citrate synthase.

Departantento de Bioquílnica* e de Clínica Médica** da Universidade Federal do Paraná (UFPR). Estudo nealizado com auxilio do Conselho Nacional de Desenvolvimento Científico e Tecnológico - CNPq (Pros. n 52.1478/96, 30.0738/ 87). Accitc: 15-janeiro-1997.

Dr. Amibal P. Campello - Depatamento de Bioquímica, UFPR - Caixa Postal 19046 - 81531-990 Cuntiba PR - Blassil. 
As doenças mitocondriais têm sido reconhecidas por muitos anos, iniciando-se com o cstudo pioneiro de Luft ct al. "2 $\mathrm{cm} \mathrm{1962,} \mathrm{quando} \mathrm{foi} \mathrm{diagnosticado} \mathrm{hipermetabolismo} \mathrm{cm}$ uma mulher, devido a deficiência no acoplamento oxidação-fosforilação, em mitocôndrias de músculo. Geralmente, pacientes com doenças mitocondriais exibem desordens nos tecidos com alta demanda acróbica, isto ć, cérebro, músculo esquelético c coração. Os sintomas são variados, com os pacientes frequentemente apresentando cardiopatias ou doenças generalizadas do músculo, fígado e/ou rins ${ }^{3}$. Defeitos na cadeia respiratória são reconhecidos como causas importantes das doenças mitocondriais. Deficiências múltiplas, bem como as deficiências isoladas dos complexos I-IV têm sido descritas ${ }^{4.6}$. A caracterização das miopatias mitocondriais por critérios morfológicos não é apropriada, por diversas razões ${ }^{5}$ : 1) anormalidades morfológicas não distinguem as diferentes miopatias mitocondriais; 2) anormalidades morfológicas das mitocôndrias não são restritas a síndromes resultantes de erros primários do metabolismo mitocondrial; 3) alteraçōes morfológiças mitocondriais são escassas em pacientes com erros definidos no metabolismo mitocondrial, tais como, deficiências da carnitina palmitoil-transferase ou deficiências da desidrogenase pirúvica. Um diagnóstico mais preciso pode ser feito por análise bioquímica dos tecidos envolvidos. A determinação das atividades enzimáticas mitocondriais em músculo esquelético humano, obtido por biópsia, pode propiciar um método alternativo de diagnóstico, preciso e específico, além de permitir caracterizá os complexos enzimáticos deficientes.

O presente estudo estabelece as atividades enzimáticas normais em um grupo controle c rclata 10 casos de pacientes cujos diagnósticos anatomopatológicos indicavam suspeitas de miopatias mitocondriais. Foram determinadas as atividades enzimáticas ligadas à cadeia respiratória: NADH desidrogenasc, NADH citocromo c redutase, succinato citocromo $\mathrm{c}$ redutase, citocromo $\mathrm{c}$ oxidase, além das enzimas citrato sintase e succinato desidrogenase, do ciclo do ácido cítrico.

\section{MATERIAIS E MÉTODOS}

Obtenças de recido muscular esquelético humano para isolamento de mitocôndrias. Amostras de quadriceps c biceps $(0,1$ a $0,4 \mathrm{~g}$ ), oriundas de pacientes de ortopedia e traumatologia (grupo controle) ou de suspeitos de miopatias mitocondriais, foram obtidas por biópsia. sob anestesia local, peridural ou raquideana. Imediatamente após a biópsia, a peça foi lavada com soro fisiológico gelado c rapidamente armazenada em nitrogênio líquido, no máximo até 7 dias após a coleta do material. A retirada de tecido muscular ocorreu imediatamente após a indução anestésica. O tempo decorrido, entre a retirada do tecido e o seu armazenamento, não ultrapassou 10 minutos. Tal procedimento visa cvitar a degradação proteolítica das enzimas. ${ }^{2 \%}$. A escolha do quadriceps ou biccps é justificada pelo fato de que, nestes músculos, há predominância de fibras do tipo I (aeróbicas) em relação ìs fibras do tipo II (anaeróbicas). Nenhum dos indivíduos (grupo controle ou doentes) estava usando qualquer tipo de medicação de uso prolongado e contínuo quando da intervenção cirúrgici.

Isolamento de mitocôndrias de muisculo esquelético humano. Mitocôndrias de músculo esquelético humano foram isoladas de acordo con o método descrito por Jung et al. *, usando-se o meio de extração contendo: D-inanitol 250,0 mM, HEPES $10,0 \mathrm{mM}$ ( $\mathrm{pH} 7,2)$, EGTA $1,0 \mathrm{mM}$ e BSA 0,1\%. O músculo (0,1 a 0,4g) foi descongelado em meio de extração gelado, finamente picado com bisturi e homogencizado em homogeneizador van Potter. $O$ homogencizado foi então centrifugado a $490 x g$ a $2^{\circ} \mathrm{C}$ por 10 minutos, a fim de climinar os restos de células intacias, membranas e núcleos. O sedimento foi desprezado e o sobrenadante foi centrifugado a $9.480 \times$ g a $2^{\circ} \mathrm{C}$ durante 15 minutos. O sedimento obtido, constituído de mitocondrias intactas, foi ressuspenso em meio de extração e armazenado em nitrogênio líquido.

Obtencião de mitocôndrias rompidas de músculo esquelético humano. Mitocôndrias intactas foram congeladas em nitrogênio líquido por aproximadamente 3 horas, descongeladas rapidamente e recongeladas; esta operaçño foi repetida 3 vezes, em intervalos de 30 minutos, sendo fulalmente conservadas em banho de gelo, durante a realização dos experimentos.

Determiną̧âo das atividades enzimáticas envolvidas no metabolismn energético. As atividades enzimáticas foram deterıninadas cm mitocôndrias rompidas utilizando-se espectrofotômetro Gilford modelo 252, acoplado al registrador: O volume final do meio de reaçäo foi sempre de $1,0 \mathrm{ml}$, utilizando-se cubetas de vidro com $1,0 \mathrm{~cm}$ de caminho óptico. A temperatura do sistema foi mantida en $28^{\circ} \mathrm{C}$ por banho-maria termostatizado. 
Citocrmmo c: oxidase (EC 1.9.3.1) - A atividade foi determinada segundo o método descrito por Masols et al. ${ }^{13}$. O sistema de reação continha: tampão fosfato $50,0 \mathrm{mM}(\mathrm{pH} \mathrm{7,4)}$, EDTA $2,0 \mathrm{mM}$, ferrocitocromo $\mathrm{c}$ $30,0 \mu \mathrm{M}$ e aproximadamente $20,0 \mu \mathrm{g}$ de proteína mitocondrial. A reação foi iniciada pela adlição de mitocôndria sendo a velocidade de oxidação do ferrocitocromo c acompanhada $\mathrm{cm} 550 \mathrm{~nm}$. A atividade específica foi expressá em nanomoles de citocromo $c$ oxidado por minuto por ing de proteína, utilizando-se o coeficientc de extinção molar do citocromo $\mathrm{c}: \mathrm{E}_{5 \mathrm{~S})}$ red-ox $=19 \cdot 10^{3} \mathrm{M}^{-1} \cdot \mathrm{cm}^{-1}$.

NADH citocromo c redutase (E.C. 1.6.99.3) - A atividade foi determinada segundo o método descrito por Somlon"1). O sistema de rcaçāo continha: tampão fosfato $50,0 \mathrm{mM}$ (pH 7,4), EDTA 2,0 mM, NADH 50,0 $\mu \mathrm{M}$, ferricitocromo c $40,0 \mu \mathrm{M}, \mathrm{NaCN}$ 1,0 mM e aproximadamente $20,0 \mu \mathrm{g}$ de proteína mitocondrial. A reaçĩo foi iniciada pela adiçāo de mitocôndria sendo a velocidade de redução do ferricitocromo c acompanhada a $5.50 \mathrm{~nm}$. A atividade específica foi expressa em nanomoles de citocromo $\mathrm{c}$ reduzido por mg de proteína, utilizando-se o coeficiente de extinção molar do citocromo $c$.

NADH desidregenase (E.C. 1.6.99.3) - A atividade fai determinada segundo o método descrito por Singer $^{1 x}$. O sistema de reação continha: tampão fosfato $50,0 \mathrm{mM}(\mathrm{pH} 7,4)$, EDTA $2,0 \mathrm{mM}, \mathrm{NADH} \mid 50,0 \mu \mathrm{M}$. ferricianeto $600,0 \mu \mathrm{M}$, rotenona $10,0 \mathrm{mM}$ e aproximadamente $20,0 \mu \mathrm{g}$ de proteína mitocondrial. A reação foi iniciada pela adição de mitocôndria sendo a velocidade de redução do ferricianeto acompanhaca a $420 \mathrm{~nm}$. A atividade específica foi expressa em nanomoles de ferricianeto reduzido por minuto por mg de proteína, utilizandose o coeficiente de extinção molar do ferricianeto: $E_{420}$ red-ox $=1040 \mathrm{M}^{-1} \cdot \mathrm{cm}^{-1}$.

Succinate citocrmno credutrse (E.C. 1.3.99.1) - A atividade foi determinada segundo o método descrito

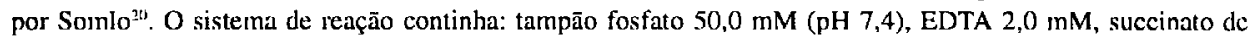
sódio $5,0 \mu \mathrm{M}, \mathrm{NaCN} 1,0 \mathrm{mM}$, rotenona $10,0 \mu \mathrm{M}$ e aproximadamente $40,0 \mu \mathrm{g}$ de proteína mitocondrial. A mistura foi incubada com succinato por 5 minutos a $28^{\circ} \mathrm{C}$ para pré-ativaçăo da enzima, sendo a ração iniciadia pcla adição do ferricitocromo c 40,0 $\mu \mathrm{M}$. A velocidade de redução do ferricitocromo $c$ foi acompanhada a 550 nm. A atividade específica foi expressa em nanomoles de citocromo c reduzido por minuto por mg de proteína. utilizando-se o coeficiente de extinção molar do citocromo $c$.

Cirrate sintase (E.C. 4.1.3.7) - A atividade foi determinada segundo o método descrito por Srewe-1. O sistema de renção continha: ácido 5,5'-ditio-bis-(2-nitrobenzóico) (DTNB) 100,0 $\mu \mathrm{M}$, acctil CoA $300,0 \mu \mathrm{M} \mathrm{e}$ aproximadamente $20,0 \mu \mathrm{g}$ de proteína mitocondrial. A absorção a $412 \mathrm{~nm}$ foi acompanhada por 3 minutos para medir a possível desacilação do acetil-CoA, sendo posteriormente a reação iniciada pela adição de $500,0 \mu \mathrm{M}$ de oxaloacetato. A velocidade de formação dos grupos SH livres correpondente ao CoA-SH e sua complexaçăo com o DTNB, gerando um íon mercaptídeo foi monitorada em $412 \mathrm{~nm}$, onde o produto formado apresenta forte absorção. A atividade específica foi expressa em nanomoles de CoA-SH formado por minuto por mg de proteína, utilizando-se o coeficiente de extinção molar do íon mercaptídeo: $\mathrm{E}_{412}=13,6.10^{3} \mathrm{M}^{-1} \cdot \mathrm{cm}^{-1}$.

Succinato desidrogenase (E.C. 1.3.99.1) - A atividade foi determinada segundo o método de Singer ${ }^{1 x}$, utilizando-se 2,6 diclorofenolindofenol (DPIP) e fenazina metasulfato (PMS) como sistema aceptor de elćtrons. O sistema de reação continha: tampão fosfato $50,0 \mathrm{mM}(\mathrm{pH} 7,4)$, EDTA $2,0 \mathrm{mM}$, succinato de sódio $20,0 \mathrm{mM}$, $\mathrm{NaCN} 1,0 \mathrm{mM}$, rotenona $10.0 \mu \mathrm{M}$ e aproximadamente $40,0 \mu \mathrm{g}$ de proteína mitocondrial. A mistura foi incubada com succinato de sódio durante 5 minutos a $28^{\circ} \mathrm{C}$ para pré-ativação da enzima, sendo a reação iniciada pela adiçāo de DPIP $60,0 \mu \mathrm{M}$ e PMS 1,0 mM. A velocidade de redução do DPIP foi acompanhađla em $600 \mathrm{~nm}$. A atividade específica foi expressa em nanomoles de DPIP reduzido por minuto por $\mathrm{mg}$ de proteína, utilizando-se o coeficiente de extinção molar de DPIP reduzido: $\mathrm{E}_{\mathrm{f}(\mathrm{x})}=19.10^{7} \mathrm{M}^{-1} \cdot \mathrm{cm}^{-1}$.

Obtençăo de ferrocitocromo c. Citocromo c bovino foi reduzido com ditionito de sódio e a solução aquosa filtrada em coluna Sephadex G-25, a fim de separar o ferrocitocromo c do excesso de ditionito ${ }^{22}$. A concentração de citocromo $c$ reduzido foi determinada espectrofotometricamente, utilizando-se o coeficiente de extiıção molar $27,7.10^{3} \mathrm{M}^{-1} \cdot \mathrm{cnt}^{-1}$ para a hemoprotcína reduzida.

Determinaçầo da concentraçẫo de proteinas As concentraçōes de proteínas das preparaçōes mitocondriais foram determinadas pelo método descrito por Lowry et al. ", utilizando-se a soroalbumina bovina como padrão.

Análise esfatistica $O$ parâmetro estatístico empregado na comparação das médias de atividades cnzimiticas entre o grupo controle e doentes foi o teste $t$ de Student, para dados não parcados, utilizando-se o nível de significância de $5 \%$. 


\section{RESULTADOS}

O grupo controle foi constituído de 13 indivíduos (9 homens c 4 mulheres) que não apresentavam docnças musculares e com idade entre 11 e 50 anos. Tratava-se de pacientes de ortopedia c traumatologia, mais especificamente com problemas de joelho (rompimento de ligamentos, luxação de patcla com cntorse) c fratura exposta de fêmur. Nestes casos, em que o tratamento cxigia intervenção cirúrgica c que o acesso permitia exposição do quadriceps, foram feitas as biópsias. Com relação ao grupo doentc, foram analisados 10 casos ( 7 homens e 3 mulheres) com idade entre $4 \mathrm{c} 50$ anos, cujo diagnóstico anatomopatológico indicava suspcita de miopatia mitocondrial.

O teste F. scguido do teste de Tukcy, aplicado ao grupo controlc, mostrou não haver diferença cstalísticia a nívcl de $5 \%$ de significância nas diferentes atividades enzimáticas. cm função dos seguintes parîmetros: scxo, idade c origem do músculo esquelético avaliado (biceps ou quadriceps).

O testc t de Student mostrou diferença cstatística significativa ( $p<0,05)$, na comparação das médias das atividades enzimáticas determinadas entre o grupo controle e cada um dos pacientes.

Nas Tabelas 1 a 6 constam as atividades enzimáticas do grupo controle e dos docntes.

A partir dos resultados aprescntados nas Tabelas 1 a 6, pode-se analisar as características do metabolismo mitocondrial de cada paciente.

lacienle EML. Apresenta diminuição da atividade das seguintes enzimas: NADH desidrogenase ( $54,7 \%$ do controle). NADH citocromo c redutase ( $43,8 \%$ do controle), succinato desidrogenase $(42,4 \%$ do controle), succinalo cilocromo c redutase ( $31,2 \%$ do controle) e citrato sintase ( $44,5 \%$ do controle). A ativirlade da citocromo oxidase foi nomal comparada ao grupo controle. Este perfil permite sugerir deficiências combinadas da cadeia respiratótia, a nível dos complexos I e II, além de provável deficiência do complexo III. Por outro lado, a deficiência na atividade da enzima citrato sintase indica possíveis anormalidades no metabolismo oxidativo a nível do cicio do ácido cítrico.

Tabela 1. Comparacaios da atividade enzimatica da NADH desidiogenase entre o grapo controle e doentes.

\begin{tabular}{cccc}
\hline dentificiçio & n & $\mathrm{A} \pm \mathrm{DP}$ & $\mathrm{p}$ \\
\hline Controle & 67 & $2416 \pm 336$ & - \\
EML & 5 & $1322 \pm 32$ & $<0,05$ \\
EST & 6 & $1806 \pm 158$ & $<0,05$ \\
GFT & 4 & $1257 \pm 16$ & $<0,05$ \\
JAS & 4 & $658 \pm 172$ & $<0,05$ \\
JNN & 7 & $1699 \pm 122$ & $<0,05$ \\
JS & 3 & $509 \pm 46$ & $<0,05$ \\
LOL & 6 & $986 \pm 83$ & $<0,05$ \\
PPN & 5 & $993 \pm 77$ & $<0,05$ \\
RF & 5 & $1727 \pm 79$ & $<0,05$ \\
ZS & 7 & $2431 \pm 121$ & $>0,05$ \\
\hline
\end{tabular}

n, número de repetiçōes: Dl, desvio pidtāo. $A$, atividade cnzimáticic específica médial (nmoles de ferricianeto reduzido/ $\min \times$ me de protcíltil).
Tabela 2. Comparaşão da abividade enzimática da NADH citocromo c redutase entre os grapo controle e donentes.

\begin{tabular}{cccc}
\hline Identificaçĩo & $\mathrm{n}$ & $\mathrm{A} \pm \mathrm{DP}$ & $\mathrm{p}$ \\
\hline Controle & 79 & $115 \pm 2.5$ & - \\
EML & 5 & $50 \pm 2$ & $<0,05$ \\
EST & 5 & $84 \pm 5$ & $<0,05$ \\
GFT & 5 & $32 \pm 2$ & $<0,05$ \\
JAS & 4 & $47 \pm 11$ & $<0,05$ \\
JNN & 5 & $62 \pm 5$ & $<0,05$ \\
JS & 4 & $11 \pm 1$ & $<0,05$ \\
LOL & 5 & $39 \pm 4$ & $<0,05$ \\
PPN & 5 & $32 \pm 3$ & $<0,05$ \\
RF & 6 & $55 \pm 3$ & $<0,05$ \\
ZS & 5 & $126 \pm 4$ & $>0,05$ \\
\hline
\end{tabular}

n. número de repetiçōes; DP. desvio pudrão: $\mathrm{A}$. ittividade enzimática específicu médial (nmoles de citocromo c reduzido/ min x mg de proteína). 
Tabela 3. Commuraşăo da atividade enzimática da succinato desidrogenase entre ogrtipo controle e doentes.

\begin{tabular}{cccc}
\hline Identificaçũo & $n$ & $\mathrm{~A} \pm \mathrm{DP}$ & $\mathrm{p}$ \\
\hline Controlc & 64 & $110 \pm 2 \mathrm{l}$ & - \\
EML & 5 & $47 \pm 3$ & $<0,05$ \\
EST & 5 & $76 \pm 5$ & $<0,05$ \\
GFT & $*$ & $*$ & $*$ \\
JAS & 3 & $29 \pm 5$ & $<0,05$ \\
JNN & 4 & $60 \pm 2$ & $<0,05$ \\
IS & 3 & $11 \pm 2$ & $<0,05$ \\
LOL & 6 & $19 \pm 4$ & $<0,05$ \\
PPN & 4 & $23 \pm 2$ & $<0,05$ \\
RF & 5 & $62 \pm 2$ & $<0,05$ \\
ZS & 6 & $63 \pm 3$ & $<0,05$
\end{tabular}

11, número de repetiçôes: DP, desvio pauluão; *, atividade enzimática não determinada; $A$, ati vidade enzimática específica média (nmoles de DPJP reduzido/min $x$ me de proteína).

Tabela 5. Comparaşāo da atividade enzimática da citocromo c oxidase entre o grupo controle e doentes.

\begin{tabular}{cccc}
\hline Identificaçĩo & $\mathrm{n}$ & $\mathrm{A} \pm \mathrm{DP}$ & $\mathrm{p}$ \\
\hline Controle & 78 & $220 \pm 65$ & - \\
EML & 5 & $267 \pm 13$ & $>0,05$ \\
EST & 5 & $136 \pm 12$ & $<0,05$ \\
GFT & 3 & $142 \pm 5$ & $<0,05$ \\
JAS & 4 & $45 \pm 4$ & $<0,05$ \\
JNN & 6 & $208 \pm 5$ & $>0,05$ \\
JS & 3 & $34 \pm 3$ & $<0,05$ \\
LOL & 5 & $118 \pm 5$ & $<0,05$ \\
PPN & 5 & $124 \pm 4$ & $<0,05$ \\
RF & 5 & $161 \pm 6$ & $<0,05$ \\
ZS & 5 & $243 \pm 8$ & $>0,05$
\end{tabular}

n. número de repetiçōes; DP. desvio padrūo; A, atividade enzinuítical especifitua média (nmoles de citocromo c oxidado/ mill $x \mathrm{mg}$ de proteína)
Tabela 4. Comparatsion da anividade enzimánica da succinato citocromo c redutase entre " grapos controle e dientes.

\begin{tabular}{cccc}
\hline Identificaçĩo & $n$ & $\mathrm{~A} \pm \mathrm{DP}$ & $\mathrm{p}$ \\
\hline Controlc & 91 & $91 \pm 13$ & - \\
EML & 5 & $28 \pm 2$ & $<0,05$ \\
EST & 5 & $54 \pm 2$ & $<0,05$ \\
GFT & 3 & $13 \pm 1$ & $<0,05$ \\
JAS & 4 & $14 \pm 3$ & $<0,05$ \\
JNN & 6 & $45 \pm 4$ & $<0,05$ \\
JS & 3 & $14 \pm 1$ & $<0,05$ \\
LOL & 6 & $22 \pm 2$ & $<0,05$ \\
PPN & 5 & $15 \pm 1$ & $<0,05$ \\
RF & 6 & $32 \pm 3$ & $<0,05$ \\
ZS & 5 & $29 \pm 3$ & $<0,05$ \\
\hline
\end{tabular}

$\mathrm{n}$, número de repetiçôs: DP. desvio padrĩo; $A$. atividade cnzimática cspecífica média (nmoles de citocromo c reduzido) min $x$ mg de proteínil).

Tabela 6. Comparaşäo da atividade enzimática da citrato sintase entre o grupo controle e doentes.

\begin{tabular}{cccc}
\hline Identificação & $\mathrm{n}$ & $\mathrm{A} \pm \mathrm{DP}$ & $\mathrm{p}$ \\
\hline Controle & 74 & $237 \pm 41$ & - \\
EML & 6 & $105 \pm 5$ & $<0,05$ \\
EST & 4 & $116 \pm 4$ & $<0,05$ \\
GFT & 3 & $82 \pm 4$ & $<0,05$ \\
JAS & 3 & $57 \pm 2$ & $<0,05$ \\
JNN & 6 & $139 \pm 5$ & $<0,05$ \\
JS & 3 & $61 \pm 1$ & $<0,05$ \\
LOL & 5 & $61 \pm 3$ & $<0,05$ \\
PPN & 4 & $88 \pm 4$ & $<0,05$ \\
RF & 6 & $125 \pm 6$ & $<0,05$ \\
ZS & 6 & $214 \pm 5$ & $>0,05$
\end{tabular}

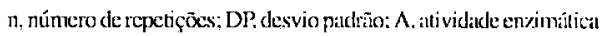
especíticu médiu (nmoles de $\mathrm{Co \Lambda}-\mathrm{SH} / \min \mathrm{x}$ mg de proteínil). 
Paciente EST. Este paciente apresentou diminuição na atividade de todas as enzimas ensaiadas, sendo o percentual de atividade remanescente, comparado ao controle, descrito a seguir: NADH desidrogenase $(74,7 \%$ do controle), NADH citocromo c redutase ( $72,8 \%$ do controle), succinato desidrogenase $(69,1 \%$ do controle), succinato citocromo $\mathrm{c}$ redutase (60\% do controle), citocromo $\mathrm{c}$ oxidase $(61,6 \%$ do controle) e citrato sintase (49\% do controle). Estes resultados sugerem múltiplas deficiências da cadeia respiratória, a nível dos complexos I, II e IV. No caso do complexo I, o grau de anormalidade foi relativamente baixo, quando comparado as demais complexos cnzimáticos afetados. Unza possível deficiência do complexo III não pode ser comprovada pelos resultados obtidos. Alćm disso, a deficiência na atividade da enzima citrato sintase indica provável anormalidade no metabolismo oxidativo a nivel do ciclo do ácido cítrico.

Paciente GFT: Este paciente apresentou diminuição na atividade de todas as enzimas ensaiadas, sendo o percentual de atividade remanescente, comparado ao controle, como mostrado a seguir: NADH desidrogenase ( $52 \%$ do controle) , NADH citocromo $\mathrm{c}$ redutase $(27,8 \%$ do controle), succinato citocromo c redutase $(13,8 \%$ do controle), citocromo c oxidase $(64,4 \%$ do controle) e citrato sintase $(34,7 \%$ do controle). Os resultados sugerem deficiências combinadas da cadeia respiratória a nível dos complexos I, III e IV. A indicação de envolvimento do complexo III advém do fato qué a atividade da NADH citocromo c redutase (complexos I + III) é significativanenté menor que a atividade enzimática da NADH desidrogenase (par1e do complexo I). A caracterização de possível disfunção a nível do complexo II, ficou prejudicada pelo fato da atividade da enzima succinato desidrogenase (parte do complexo II) não ter sido determinada. Além disso, a deficiência na atividade cla enzina citrato sintase indica provâvel anormalidade no metabolismo oxidativo a nivel do ciclo do ácido cítrico.

Prciente JAS. Este paciente apresentou diminuição na atividade de todas as enzimas ensaiadas, sendo o percentual de atividade remanescente, comparado ao controle, como mostrado a seguir: NADH desidrogenase (27,2\% do controle), NADH citocromo c redutase $(40,8 \%$ do controle), succinato desidrogenase $(25,9 \%$ do controle), succinato citocromo c redutase ( $15,8 \%$ do controle), citocromo c oxidase $(20,5 \%$ do controle) e citrato sintase (24.1\% do controle). Observa-se severa redução em todas as atividades enzimáticas, revelando sério comprometimento do metabolismo cnergético. É possível sugerir a existência de múltiplas deficiências na cadeia Icspintória, a nivel clos complexos I, II, e IV. A indicação de deficiência a nível do complexo III não posle ser comprovada pelos resultados obtidos. Além disso, a deficiência na atividade da enziına citrato sintase indica provível anormalidarle no metabolisıno oxidativo a nível do ciclo do ácido cítrico.

Puciente JNN. O paciente apresentou diminuiçăo na atividade das várias enzimas, sendo o percentual de atividade remanescente, comparado ao controle, conforme descrito a seguir: NADH desidrogenase (70\% do controle), NADH citocromo c redutase $(54,1 \%$ do controle), succinato desidrogenase $(54,5 \%$ do controle), succinato citocromo c redutase ( $50 \%$ do controle) e citrato sintase $(58,9 \%$ do controle). A atividade da enzima citocromo $c$ oxiclase $(94,7 \%$ do controle) mostrou resultado normal. Este perfil permite sugerir deficiências combinadas da cadeia respiratória, a nível dos complexos I e Il. A indicação de possível defíciência no complexo III não pode ser comprovada pelos resultados apresentados. Além disso, a deficiência na atividade da enzima citrato sintasc indica provável anormaljdade no metabolismo oxidlativo a nível clo ciclo do áciclo cítrico. Devese ressaltar também que a caracterização bioquímica deste paciente foi similar à obtida para o paciente EML, ambos apresentando deficiências em todas as enzimas ensaiadas, com exceção da citocromo c oxidasc.

Paciente IS. O paciente apresentou diminuição nas atividades de todas as enzimas ensaiadas, sendo o percentual dic atividade remanescente, comparado ao controle, como descrito a seguir: NADH desidrogenase (21,1\% do controle), NADH citocromo c redutase $(9,3 \%$ do controle), succinato desidrogenase $(10,2 \%$ do controle), succinato citocromo c redutase ( $15,9 \%$ do controle), citocromo c oxidase (I5,3\% do controle) e citrato sintase ( $26 \%$ do controle). A partir destes resultados, fica evidente o sério comprometimento da função mitocondrial neste paciente. Este perfil evidencia a possibilidade de múltiplas cleficiências da cadcia respiratória, a nível dos complexos 1, II, IIl e IV. Além disso, a deficiência na atividade da enzima citrato sintase indica provável anormalidade no metabolismo oxidativo a nível do ciclo do ácido cítrico.

Paciente LOL. O paciente apresentou diminuição nas atividades de todas as enzimas ensaiadas, sendo o percentual de atividade remanescente, comparado ao controle, como descrito a seguir: NADH desidrogenase 
(40,8\% do controle), NADH citocromo c redutase ( $34 \%$ do controle), succinato desidrogenase ( $17,2 \%$ do controle), succinato citocromo $\mathrm{c}$ redutase $(24,6 \%$ do controle), citocromo c oxidase $(53,8 \%$ do controle) e citrato sintase (25,7\% do controle). Este perfil permite sugerir deficiências múltiplas na cadeia respiratória, a nível dos complexos I, II, III c IV. Além disso, a deficiência na atividade da enzima citrato sintase indica provável anormalidade no metabolismo oxidativo a nível do ciclo do ácido cítrico.

Pecciente PPN. A paciente apresentou diminuição nas atividades de todas as enzimas ensaiadas, sendo o percentual de atividade remanescente, comparado a controle, como descrito a seguir: NADH desidrogenase ( $41,1 \%$ do controle), NADH citocromo c redutase ( $28 \%$ do controle), succinato desidrogenase ( $20,4 \%$ do controlc). succinato citocromo $\mathrm{c}$ redutase $(16,6 \%$ do controle), citocromo $\mathrm{c}$ oxidase $(56,3 \%$ do controle) e citrato sintase ( $37,1 \%$ do controle). A partir de tais resultados, fica evidente o sério comprometimento da função mitocondrial neste paciente. Este perfil permite sugerir múltiplas deficiências na cadeia respiratória, a nível dos complexos I, II, IIl e IV. Além disso, a deficiência na atividade da enzima citrato sintase indica provável anormalidade no metabolismo oxidativo a nível do ciclo do ácido cítrico.

Paciente RF: O paciente apresentou diminuiçän nas atividades de todas as enzimas ensaiadas, sendo o percentual de atividade remanescente, comparado ao controle, como descrito a seguir: NADH desidrogenase ( $71,5 \%$ do controle), NADH citociomo c redutase $(48,2 \%$ do controle), succinato desidrogenase $(56,1 \%$ do controle), succinato citocromo $\mathrm{c}$ redutase ( $35 \%$ do controle), citocromo c oxidase $(73,1 \%$ do controle) e citrato sintase ( $52,7 \%$ do controle). Este perfil permite sugerir múltiplas deficiências da cadeia respiratória, a nível dos complexos 1, Il, Ill e IV. A indicaçâo de provável envolvimento do complexo III advém do fato que a atividade da NADH citocromo c redutase (complexos I + III) ć significativamente menor que a atividade enzimática cla NADH desidrogenase (parte do complexo I). Além disso, a deficiência na atividade da enzima citrato sintase indica provável anormalidade no metabolismo oxidativo a nível do ciclo do ácido cítrico.

Pecciente ZS. A paciente apresentou diminuição nas atividades de apenas duas enzimas, sendo o percentual de atividade remanescente, comparado ao controle, como descrito a seguir: succinato desidrogenase $\langle 57,5 \%$ do controle) e succinato citocromo c redutase (32,5\% do controle). As atividades das demais enzimas investigadas, mostraram-se absolutamente normais. Este perfil permite caracterizar deficiência isolada na cadeia respiratória. a nível do complexo II. Dentre os 10 casos analisados este foi o único em que a disfunção mitocondrial mantevese restrita a apenas um complexo respiratório, no qual segundo a literatura ${ }^{1+16}$ anomalidades são menos frequentes.

\section{DISCUSSĀO}

Alguns pesquisadores têm demonstrado especial preocupação com relação aos cuidados c critérios necessários na condução dos estudos bioquímicos sobrc doenças mitocondriais. Estes incluem: seleção de grupo controle apropriado, condições ideais de realização da biópsia c armazenamento da amostra coletada e emprego de metodologia adequada para isolamento c determinação das respectivas atividades enzimáticas. Muitas vezes, a diversidade e a complexidade dos resultados bioquímicos encontrados para uma mesma síndrome clínica, devem-se às diferenças nas técnicas experimentais adotadas.

Na seleção do grupo controle, foram observadas todas as recomendações citadas na literatura. É fundamental que a performance oxidativa das mitocôndrias dos doentes seja comparada com controles apropriados ${ }^{17}$. Por isso, indivíduos com padrão de atividade física semelhante, foram escolhidos para a composição do grupo controle, evitando-se sempre os esportistas.

Os pré-requisitos necessários para que os indivíduos pudessem compor o grupo controle foram: 1) não serem portadores de patologia neuromuscular; 2) não estarem fazendo uso prolongado ou contínuo de fármacos; 3) as cirurgias para correção dos problemas ortopédicos ou de fraturas apresentados pelos pacientes fossem realizadas com anestesia de bloqueio parcial (local, peridural ou raquianestesia), compatíveis com o tipo de anestesia exigido pelo estudo; 4) o acesso cirúrgico 
para correçāo do problema apresentado pelo paciente permitisse a cxposição do quadriceps ou biceps, músculos padronizados para o presente estudo.

Especificamente, com relaçāo ao tipo de anestesia, Yorifugi et al. ${ }^{23} \mathrm{e}$ Zheng $\mathrm{cl}$ al. ${ }^{24}$ discutiram a inviabilidade da realização da biópsia muscular por anestesia geral em tais estudos, mostrando que as atividades cnzimáticass $\mathrm{cm}$ mitocôndrias assim isoladas apresentam valores significativamente mais baixos c com grande dispcrsão, em comparação às atividades enzimáticas determinadas em mitocôncirias de músculo obtidas por biópsia com anestesia de bloqueio parcial.

Com relação ao isolamento c obtenção dc mitocôndrias, levou-se em consideração que o músculo csquclético contém concentração relativamente alta de $\mathrm{Ca}^{2+}$, o qual pode danificar a mitocôndria durantc o isolamento ${ }^{10}$. Para evitar o possível efeito deletério deste íon, a composição dos meios de extração c reação usados continham respectivamente, EGTA c EDTA, conhecidos quelantes de cálcio c ions divalentes.

As mitocôndrias loram rompidas por congelamento e descongelamento em um ciclo de três vezes, conforme preconizado por vários autores ${ }^{2.724}$. Este tratamento é necessário, pois mitocôndrias intactas não săo permeáveis a substratos como o NADH e outros aceitadores/doadores de elétrons utilizados nos ensaios espectrofotométricos. Além disso, este tratamento evita trauma mecânico à organcla, que podcria interfcrir na atividade dos complexos enzimáticos da cadeia respiratória. Em especial, o complexo I tem sido considerado menos cstável do que o complexo $\mathrm{II}^{14} \mathrm{c}$ altamente suscetível a mudanças lísicas proporcionadas por stress mecânico ${ }^{17}$. Quanto à cnzima citocromo c oxidase, tem sido descrita como a mais lábil dentre as cnzimas da cadcia respiratória scndo o rompimento mecânico prejudicial à atividade do complexo $0^{2+}$. De acordo com Singer ct al. ${ }^{13}$, o crro mais comumente encontrado nos ensaios com a succinato desidrogenase e succinato citocromo c redutase é a falta de pré-alivação das enzimas com o próprio substrato (succinato). A pré-ativação é necessária para remover a presença de quantidades variáveis de oxaloacetato, um inibidor competitivo das enzimas.

A investigąção bioquímica rcalizada neste estudo, revelou-se adequada para o estabelecimento de diagnóstico nosológico preciso c facilmente aplicável aos objetivos clínicos. Os resultados possibilitam ainda a localização de deficiências enzimáticas específicas do mctabolismo mitocondrial.

\section{REFERÊNCIAS}

1. Behbehani AW. Gowbel H. Osse G. Gabriel M, Langenbeck U, Berden I, Berger R, Schutgens RuH. Mitochondrial myopathy with latic alcidosis and deficient activity of muscle succinate cytocbrome-c-oxidoreductase. Eur I Pediatr. 1984;143:67-71.

2. BindolT 1 A. Desmelle C. Birch-Machin MA. Pellissier JF, Serratrice G, Dravet C. Bureau M. Howell N. Turnbull DM. Multiple deliects of the mitochondrial respiratory chain in a mitochondrial encephalopathy (MERRF): a clinical, biochemical. and molecular study. J Neuro! Sci. 199]: 102:17-24

3. Capaldi RA. Mitochondrial nyopithies and respiratory chain proteins. TIBS 1988;13:144-148.

4. Cooper JM. Hayes D. . Chaliss RA.I, Morgan-hughes JA, Clarck JB. Treatment of experinental NADH ubiquinone reductise deficiency with mentadione. Barain 1992;1 15:991-1000.

5. Di Mauro S. Bonilli E, Zeviani M. Nakigawa M. De Vivo D. Mitochondrial myopathies. Ann Neurol. 1985:17:521-538.

6. Hall RE: Henrikson KG. Lewis SF: Haller RG, Ketnaway NG. Mitochondrial nyopathy with succinate dehydrogenase and aconilitic deliciency: abnormalitics of scvelal iron-sulfur proteins. J Clin Invest, 1993;92:2660-2666.

7. Iehiki T. 'Tanakil M. Nishirimi M. Suziki H. Ozawa T, Kobayashi M, Wada Y. Deficicney ol subunits of complex I and milochondrial cueeplatomyopathy. Ann Neurol 1988;23:287-294.

8. Jung K. Reinholdt C. Schoie D. Inhibited efticiency of kidney mitochondria isolated fiom rats tratted will ciclosporin $\Lambda$. Nephron 1987:45:43-45.

9. Kim S.I. Lee KO. Takimiya S. Cipaldi RA. Mitochondrial myopathy involving ubiquinol-cytochrome c oxidoreductalse (complex III) identilied by immunoelectron microscopy. Biochim Biophys Actiః 1987;92:270-276.

10. Lee CP. Biochemicial studies ol isolated mitochondria from normal ind diseased tissues. Biochim Biophys Acta 1995:1271:21-28.

11. Lowry OH. Rosebrough N., F Fur AC. Randall RJ. Protein melsurement with the Folin phenol reigemt. J 13iol Chem 1951:193:265-275.

12. Luft R. Ikkos D. l'almieri G. Ernsster L. Afzelius B. A case of severe hypermetalbolism of nontlyyroid origin with a defect in the mintenane of mitoclondrial respiritory control: a correlated clinical, biochemical. and morphological study. I Clin Invesl 1962:11:1776-1804. 
13. Matson TL. Poyton RO, Whatton DC, Shatz G. Cytochtome c oxidase from bakers yeast. J Biol Chem 1973;248:1346-1354.

14. Riggx JE, Schochet SS Jr.. Fukudej AV, Papadimitriou A, Di Mauro S, Crosby TW, Gutmann L, Moxley III RT. Mitochondrial encephalomyopathy with decreased succinate-cytochrome c reductase activity. Neurology 1984:34:48-53.

15. Rivner MH, Shımsni 1 M, Hommes FA. Keirns-Sayre syndrome and complex II deficiency. Neurology 1989;39:693-696.

16. Schapiru AHV, Cooper JM, Morgan-Hughes JA, Landon DN, Clark JB. Mitochondrial myopathy with a detect of mitochondrialprotein transport. N Engl J Med 1990;323:37-42.

17. Scholte HR. Agsteribbe E, Busch HFM, Hoogenraad TU, Jennekens FGI, Van Linge B, Luyt-Houwen IEM, Ross JD, Riters MHJ, Verduin MHM. Oxidative phosphorylation in human muscle in patients with ocular myopathy and ufter general anuestliesiat. Biochin Biophys Acti 1990;1018:211-216.

18. Singer TP. Ramsay RR, Ackrell BAC. Deficiencies of NADH and succinate dehydrogenases in degenerative diseases and myopithies. Biochim Biophys Actid 1995;1271:211-219.

19. Singer TP. Determination of the activity of succinate, NADH, choline and a-glycerophosphate dehydrogenase. Methods Biochem Anal 1974:22:125-175.

20. Somlo M. Induction des lactico-citochrome c redutuses (D- et L-) de la levure aerobie pur des lactutes (D- et L-). Biochim Biophys Actu 1965;97:183-201.

21. Srere PA. Citrate synthise. Methods Enzymol 1969;13:3-11.

22. Yonetani T, Ray GS. Kinctics of the aerobic oxidation of ferrocytochrome c by cytochrome c oxidase. J Biol Chem 1965:240:3392-3398.

23. Yorifuji S. Ogasilhara S, Takahashi M, Turui S. Decreased activities in mitochondrial inner membrane electron transport system in nuscle tiom putients witl Kearns-Suyre syndrome. J Neurol 1985;71:65-75.

24. Zheng X, Shoffner JM, Voljavec $\Lambda S$, Wallace DC. Evaluation of procedures for assaying oxidative phosphorylation enzyme activitics in mitochondrial myopathy muscle biopsies. Biochim Biophys Acta. 1990.1019:1-10. 